\title{
Rankings Universitários: Análise dos Indicadores Utilizados
}

\author{
University Rankings: Analysis of Indicators
}

\author{
Sandra Mara Iesbik Valmorbida \\ Doutoranda em Contabilidade da Universidade Federal de Santa Catarina \\ Professora da Universidade Tecnológica Federal do Paraná. \\ Campus Reitor João David Ferreira Lima, Florianópolis/SC, Brasil, CEP: 88040-900. \\ smiesbik@gmail.com \\ Thuine Lopes Cardoso \\ Mestranda em Contabilidade da Universidade Federal de Santa Catarina. \\ Campus Reitor João David Ferreira Lima, Florianópolis/SC, Brasil, CEP: 88040-900. \\ thuinecardoso@hotmail.com \\ Sandra Rolim Ensslin \\ Doutora em Engenharia de Produção da Universidade Federal de Santa Catarina \\ Professora da Universidade Federal de Santa Catarina. \\ Campus Reitor João David Ferreira Lima, Florianópolis/SC, Brasil, CEP: 88040-900 \\ sensslin@gmail.com
}

\begin{abstract}
Resumo
Os rankings universitários mundiais classificam as universidades com base em um conjunto de indicadores. Entretanto, cada sistema de ranking faz uso de critérios e métricas de mensuração definidos segundo o propósito para o qual foi criado. Assim, o presente trabalho tem como objetivo identificar os indicadores apontados pelas publicações internacionais sobre "rankings universitários". A seleção dos trabalhos foi realizada por meio do instrumento de intervenção Knowledge Development Process-Constructivist (ProKnow-C) que identificou 32 artigos, publicados nas bases de dados Web of Science e Scopus e disponíveis gratuitamente na internet, alinhados ao tema, e, assim, compuseram o Portfólio Bibliográfico (PB). Com base na análise do PB, constatou-se a existência de 19 rankings universitários. Os indicadores mais frequentes estão relacionados com a infraestrutura e reputação da universidade e fazem uso de informações bibliométricas, tais como artigos publicados, número de citações de artigos e de pesquisadores e artigos indexados nas bases de dados. Constatou-se também que, há muitas diferenças de métricas utilizadas pelos rankings. No entanto, cabe salientar que, a partir da finalidade dos rankings, devem ser construídos os indicadores correlacionados com as necessidades de informação dos usuários para o(s) qual(quais) o ranking foi idealizado. O desenvolvimento de uma metodologia capaz de 'lidar' com indicadores e métricas de mensuração que neutralizem a característica de 'estágio de maturidade' das universidades configura-se como uma das possíveis contribuições para o avanço científico nesta área de conhecimento.
\end{abstract}

Palavras-chave: Rankings universitários. Indicadores. Métricas. Desempenho.

\footnotetext{
Abstract

Artigo publicado anteriormente nos Anais do $1^{\circ}$ Congresso Internacional de Métodos e Gestão em Avaliação Educacional-CIMGAE, em 2015.

Artigo submetido em maio de 2015 e aceito em agosto de 2015 pela editora Fernanda Sauerbronn, após processo de double blind review.
} 
The worldwide university rankings rank universities based on a set of indicators. However, each ranking system makes use of criteria and metrics of measurement defined according the purpose for which it was created. Thus, this paper aims to identify the indicators presented in the international literature on "university rankings". The selection of papers was conducted by the intervention instrument called Knowledge Development Process- Constructivist (ProKnow-C) which identified 32 articles published in the Web of Science and Scopus databases and freely available on the internet, aligned to the subject, and thus, composed the Bibliographical Portfolio (BP). Based on the analysis of the BP, it was found the existence of 19 university rankings. The most common indicators are related to infrastructure and reputation of the university and make use of bibliometric information, such as published articles, number of citations of articles and researchers and articles indexed in databases. It was also found that there are many different metrics used by rankings, however it should be noted that, from the purpose of the rankings, the indicators shall be constructed: the ones correlated with the users' information needs for which the ranking was designed.

Key Words: University Rankings. Indicators. Metrics. Performance.

\section{Introdução}

Os rankings universitários, amplamente divulgados, são listas de instituições que, comparativamente em ordem decrescente, apresentam melhor desempenho, seguidos dos que apresentam desempenho inferior, nos quesitos analisados (SEOANE, 2009). Trata-se de tentativas de classificar as instituições acadêmicas e de pesquisa em todo o mundo (IOANNIDIS et al., 2007).

Dessa forma, é cada vez mais frequente, por parte das universidades, a incorporação dos resultados dos rankings na promoção de mudanças em seus modelos de gestão (CHARON; WAUTERS, 2008; MARGINSON, 2014). Essas listas classificatórias influenciam a decisão de futuros alunos a ingressar ou não na universidade, nas ações de líderes executivos universitários, professores acadêmicos, governos e investidores no ensino superior (IOANNIDIS et al., 2007; CHARON; WAUTERS, 2008; MARGINSON, 2014), bem como, na definição de políticas públicas educacionais (ERKKILÄ, 2014).

Assim, percebe-se a importância e a utilidade dos rankings universitários. Tendo em vista que, um dos objetivos principais dessa classificação é avaliar a qualidade das instituições de ensino superior e de pesquisa (LUKMAN; KRAJNC; GLAVIČ, 2010), cada vez mais os países e as universidades os integram nos seus processos de avaliação de desempenho (SEOANE, 2009; CHARON; WAUTERS, 2008; MARGINSON, 2014). Há muitas críticas sobre as metodologias de classificação dos rankings no sentido de que elas podem esconder uma série de problemas metodológicos e anomalias em relação aos indicadores, às definições arbitrárias e às possibilidades de manipulação (LUKMAN; KRAJNC; GLAVIČ, 2010; CHARON; WAUTERS, 2008). Contudo, se a posição no ranking interfere na atividade universitária, deve-se destacar a importância da qualidade dos dados mensurados por ele (CHARON; WAUTERS, 2008; SEOANE, 2009; AGUILLO et al., 2010; JEREMIC; MILENKOVIC, 2014), bem como, a análise dos indicadores que responderão pela classificação divulgada.

Nesse contexto, emerge a pergunta que orienta este trabalho: Quais são os indicadores utilizados pelos principais rankings universitários mundiais? De modo a responder o problema de pesquisa, tem-se como objetivo identificar os indicadores apontados pelas publicações internacionais sobre o tema "rankings universitários". Para conduzir o processo de seleção das publicações, adotou-se o Knowledge Development Process - Construtivist (Proknow-C) como instrumento de intervenção. 
Justifica-se a realização desta pesquisa devido à oferta à comunidade científica e prática, em um único trabalho, dos indicadores extraídos de um conjunto de obras internacionais reconhecidas cientificamente e relevantes para esclarecer aos interessados os indicadores bases que informam os rankings, ainda por que atende à proposta de Buela-Casal et al. (2007) e Jeremic e Milenkovic (2014) quando apontam a necessidade de se estudar o tema. Além desta seção, este artigo apresenta, na seção 2, uma breve exposição sobre o tema central; a metodologia na seção 3; na seção 4 apresentação e discussão dos resultados; e, por fim, são apresentadas as considerações finais e as referências utilizadas.

\section{Referencial Teórico}

\subsection{Rankings Universitários}

Os rankings têm várias finalidades, entre elas está a de responder às demandas dos usuários por informações de fácil interpretação, estímular a concorrência e possibilitar melhor compreensão dos diferentes tipos de instituições. Entre os diversos tipos de ranking, os rankings universitários são muito atraentes, considerando que proporcionam uma base comparativa que permite situar as universidades no contexto nacional e até mesmo no mundial (BENITO; ROMERA, 2011).

Os rankings acadêmicos são uma tendência nos tempos atuais e, cada vez mais, diferentes partes interessadas os utilizam para obter informações sobre o desempenho das universidades (BERNARDINO; MARQUES, 2010). Esse fenômeno dos rankings tem influenciado profundamente todos os sistemas universitários, mesmo que não tenham como foco principal estabelecer um quadro competitivo entre as universidades, eles acabam fazendo isso (ROBINSON-GARCÍA et al., 2014). Tal fato acontece porque os rankings promovem "publicidade gratuita", possibilitando o reconhecimento das universidades com melhores desempenhos pelos interessados (BERNARDINO; MARQUES, 2010).

Essa publicidade decorrente dos rankings torna-se um diferencial não apenas para o recrutamento de alunos, mas também em questões como a atribuição do orçamento do governo para o ensino superior (HUANG, 2012). De acordo com Pusser e Marginson (2013), os rankings servem como uma "lente" para o estudo da situação do ensino superior. Assim, eles são usados para conferir prestígio às universidades e são úteis na alocação de recursos.

Percebe-se, assim, a abrangência, a importância e a utilidade das informações geradas pelos rankings universitários. Entretanto, cada sistema de ranking faz uso de critérios e métricas de mensuração definidos, segundo o propósito para o qual foi criado. Adicionalmente, fazem uso de diferentes metodologias que, consequentemente, demonstram resultados divergentes (JEREMIC; MILENKOVIC, 2014). Sendo assim, os indicadores são o ponto central desses sistemas. Por isso, faz-se necessário conhecer os indicadores dos rankings universitários a fim de apoiar sua escolha e utilização.

Esses indicadores permitem uma comparação entre o desempenho das universidades (BENITO; ROMERA, 2011). Uma das principais ideias, por trás do uso de indicadores, é discutir as qualidades de instituições de ensino superior de forma mais abrangente e comparável em relação às descrições dos cursos, à produção de artigos em revistas ou à qualidade de ensino (LINDBLAD, 2008). Assim, como os indicadores avaliam o desempenho de cada universidade e fornecem condições para comparação, tornam-se a base para a construção de rankings, em que qualquer indicador adotado tem grande influência sobre os resultados apresentados (HUANG, 2012). Porém ainda não existe um consenso sobre quais indicadores representam melhor a natureza e a qualidade das universidades, o que põe à prova a confiabilidade dos resultados dos rankings (ROBINSON-GARCÍA et al., 2014).

\subsection{Indicadores de Desempenho}

Valmorbida, S. M. I.; Cardoso, T. L.; Ensslin, S. R. 
Os indicadores servem para avaliar desempenhos das instituições (BENITO; ROMERA, 2011). Para cumprirem sua função, devem (i) reconhecer e valorizar os diferentes grupos de interessados (AMSLER, 2013; BENITO; ROMERA, 2011; BORNMANN; ANEGÕN; MUTZ, 2013; LIN; HUANG; CHEN, 2013); (ii) permitir gestão e controle pelas lógicas intelectuais e políticas dos interessados (AMSLER, 2013); (iii) utilizar multicritérios (BENITO; ROMERA, 2011; MARGINSON, 2014); (iv) levar em conta a missão e o objetivo da instituição (BORNMANN; ANEGÕN; MUTZ, 2013; LIN; HUANG; CHEN, 2013); (v) refletir as exigências/expectativas dos interessados e produzir informação válida sobre o desempenho das instituições (BENITO; ROMERA, 2011; BUELA-CASAL et al.; 2007; LIN; HUANG; CHEN, 2013; LINDBLAD, 2008; SEOANE, 2009; ROBINSON, 2013); (vi) permitir embasamento para tomada de decisões (KEHM, 2014; LINDBLAD, 2008); (vii) garantir a transparência e confiabilidade de seus resultados (FLORIAN, 2007; SAISANA; D'HOMBRES; SALTELLI, 2011; TURNER, 2013); (viii) promover feedback (conhecer os pontos fortes e fracos a fim de melhorar o desempenho) (BUELA-CASAL et al.; 2007; ERKKILÄ, 2014; HSIEH et al., 2012; LINDBLAD, 2008; RODIONOV; RUDSKAIA; KUSHNEVA, 2014; BOULTON, 2011); e (ix) estabelecer metas para melhoria (ERKKILÄ, 2014; HSIEH et al., 2012).

No entanto, observou-se, na literatura consultada, algumas críticas aos indicadores usados pelos rankings universitários, quais sejam: são fortemente baseados em dados bibliométricos (AGUILLO et al., 2010; AMSLER, 2013; BORNMANN; MUTZ; DANIEL, 2013; HUANG, 2012); são tendenciosos (AGUILLO et al., 2010; LINDBLAD, 2008); não são transparentes (BENITO; ROMERA, 2011; FLORIAN, 2007); não são representativos, sendo usados para uma pluralidade de cenários (AGUILLO et al., 2010; BENITO; ROMERA, 2011; JEREMIC; MILENKOVIC, 2014; apresentam arbitrariedade na atribuição de pesos dos indicadores (BENITO; ROMERA, 2011; BUELA-CASAL et al.; 2007; FLORIAN, 2007); são irreproduzíveis (FLORIAN, 2007); ocorrem mudanças nos indicadores ao longo do tempo (BOWMAN; BASTEDO, 2011); fazem uso inadequado de metodologias e ferramentas estatísticas (BUELA-CASAL et al.; 2007; SAISANA; D'HOMBRES; SALTELLI, 2011); comparam instituições que não podem ser comparáveis, porque têm missões e contextos diferentes (BENITO; ROMERA, 2011; BOULTON, 2011; BUELACASAL et al.; 2007); e não atendem aos objetivos do ranking (AGUILLO et al., 2010; BENITO; ROMERA, 2011; KEHM, 2014).

Conforme aponta Benito e Romera (2011, p. 19) a "seleção dos indicadores e a atribuição de pesos são um dos aspectos mais importantes das metodologias de ranqueamento. Para isso, devem ter base científica, confiabilidade e validade. A coleta dos dados deve ser coerente, pública e transparente para evitar erros estatísticos. A atribuição de pesos deve aparecer com base nas conclusões de extensa análise da contribuição de cada indicador para o desempenho da instituição" (traduzido pelas autoras).

\section{Metodologia da Pesquisa}

\subsection{Enquadramento Metodológico}

Esta pesquisa é de natureza descritiva, pois busca identificar os indicadores utilizados pelos rankings universitários nos artigos internacionais.

No que tange à coleta de dados, fez-se uso de dados primários e secundários. A etapa de seleção do Portfólio Bibliográfico fez uso de dados primários, uma vez que as delimitações são feitas pelos pesquisadores/participantes em todas as escolhas demandadas durante o processo. Já a etapa de identificação dos indicadores fez uso de dados secundários, uma vez que as informações são buscadas no PB. Sendo assim, a presença da subjetividade é elemento intrínseco nesse processo. Considerando que os pesquisadores também atuam/respondem 
como os participantes para evolução do processo, ou seja, os pesquisadores, durante todo o processo de desenvolvimento da primeira etapa do Proknow- $C$, necessitam apresentar suas delimitações e fazerem escolhas para que o processo evolua, essa investigação, quanto ao tipo de estratégia, faz uso da pesquisa bibliográfica e da pesquisa-ação (CRESWELL, 2014).

Quanto aos métodos específicos de investigação a coleta de dados é realizada por meio do desenvolvimento do processo estruturado Knowledge Development Process - Construtivist (ProKnow-C), que será apresentado na subseção 3.2 e resulta na identificação do Portfólio Bibliográfico.

\subsection{Procedimentos para coleta e análise dos dados}

Para seleção do material científico que será utilizado para geração de conhecimento e informações para analisar os indicadores dos rankings universitários, será utilizado o instrumento Proknow-C (Knowledge Development Process-Constructivist), desenvolvido pelo Laboratório de Metodologias Multicritério de Apoio à Decisão (LabMCDA) da Universidade Federal de Santa Catarina (UFSC) e já utilizado em várias publicações científicas (DUTRA et al., 2015; ENSSLIN et al., 2014a,b; SILVA et al., 2014; ENSSLIN; ENSSLIN; PINTO, 2013; LACERDA; ENSSLIN; ENSSLIN, 2012; ROSA et al., 2011; TASCA et al., 2010) que investigaram contextos diferentes deste.

O objetivo principal do ProKnow-C é construir conhecimento sobre um fragmento da literatura científica publicada inicialmente no pesquisador e, quando da publicação dos resultados da investigação, nos leitores da comunidade científica do tema. Para tal, o pesquisador deve realizar estas quatro etapas: (a) seleção de um Portfólio Bibliográfico (PB) de artigos sobre o fragmento que representa o tema da pesquisa; (b) análise bibliométrica do PB; (c) análise sistêmica do PB; e (d) identificação de lacunas e oportunidades de futuras pesquisas. Cada etapa demanda participação do pesquisador para sua realização. Dessa forma, o processo construtivista ocorre e evolui com base nos interesses e delimitações do pesquisador (DUTRA et al., 2015; ENSSLIN et al., 2014b; SILVA et al., 2014). Conforme demonstrado na Figura 1. 


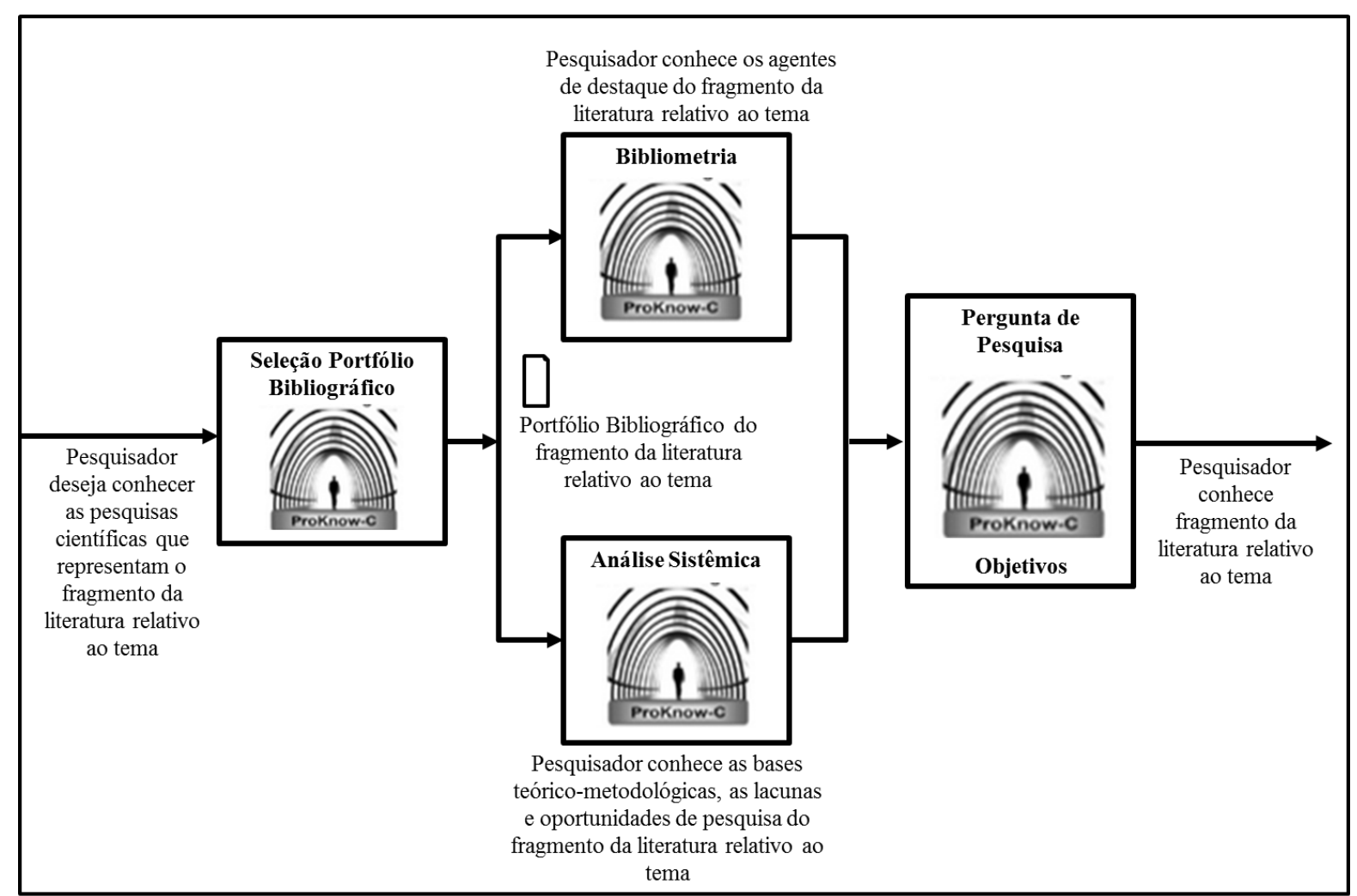

Figura 1: Etapas do Knowledge Development Process-Constructivist

Fonte: DUTRA et al. (2015); ENSSLIN et al. (2014b); SILVA et al. (2014).

Para alcance do objetivo desta pesquisa apenas a realização dessa primeira etapa faz-se necessária. A formação do Portfólio Bibliográfico (PB) de artigos que representam o tema da pesquisa é definido no Proknow-C como um "conjunto restrito de artigos científicos e relevantes" (ENSSLIN et al., 2014b, p. 593). Para isso, o processo divide-se em duas fases principais, conforme demonstrado no Quadro 1.

Quadro 1 - Fases do ProKnow-C e Resultados Encontrados

\begin{tabular}{|c|c|}
\hline Fases do ProKnow- $C$ & Resultado encontrado \\
\hline (i) seleção do banco de artigos bruto & $\begin{array}{l}\text { Bases: Web of Science e Scopus } \\
\text { Palavras-chave: "university" AND "ranking", } \\
\text { Delimitação: tipo da publicação (tipo Journal Article) e o } \\
\text { idioma do artigo (apenas em língua inglesa), sem limite } \\
\text { temporal; } \\
\text { Consulta: janeiro de } 2015 \text {; } \\
\text { Resultado: } 1.103 \text { artigos na Web of Science, e } 3.668 \text { artigos } \\
\text { na base Scopus, totalizando } 4.771 \text { artigos; }\end{array}$ \\
\hline $\begin{array}{l}\text { (b) alinhamento pela leitura do título; } \\
\text { (c) alinhamento quanto ao reconhecimento } \\
\text { científico; } \\
\text { (d) alinhamento pela leitura do resumo; e } \\
\text { (e) alinhamento pela leitura integral. }\end{array}$ & $\begin{array}{l}\text { a) publicações duplicadas, oriundas de conferências, de } \\
\text { livros, de capítulos de livros ou patentes ou editoriais } \\
\text { foram excluídas; } \\
\text { b) restaram } 175 \text { artigos; } \\
\text { c) } 52 \text { eliminados indisponíveis gratuitamente, resultando } \\
\text { em } 71 \text { artigos no banco de artigos; } \\
\text { d) } 32 \text { com resumos alinhados } \\
\text { e) } 32 \text { artigos estavam totalmente alinhados. }\end{array}$ \\
\hline
\end{tabular}

Fonte: Elaborado pelas autoras (2015). 
Cabe salientar que, o reconhecimento científico dos 71 artigos foi aferido pelas citações em outros trabalhos científicos, desde sua publicação, por meio da consulta na ferramenta Google Scholar (2015). Por meio dessa consulta, constatou-se que todos os artigos apresentam reconhecimento científico, sendo "Academic quality, league tables, and public policy: A cross-national analysis of university ranking systems" o mais reconhecido com 380 citações. Como o reconhecimento científico foi aferido em todos os artigos, o processo continuou com os 71, em que foi realizada a filtragem dos artigos selecionados pela leitura dos resumos e constatou-se que, apenas 32 artigos estavam alinhados ao tema desta pesquisa, ou seja, a evolução de rankings universitários. Esses 32 artigos formam o conjunto que os pesquisadores consideram relevantes, alinhados com a pesquisa, os quais se denominam Portfólio Bibliográfico (PB). Assim, a coleta de dados ocorrerá exatamente nesse PB; o conhecimento nos pesquisadores e os resultados serão informados por esse PB. As bases dos rankings universitários mundiais são, neste artigo, delimitadas à identificação dos indicadores utilizados por esses rankings. Foram mapeados os 32 artigos do Portfólio, sendo que, as informações foram registradas em uma planilha eletrônica, organizadas por rankings apontados pela literatura e identificados os trabalhos que expunham os indicadores utilizados e posteriormente agrupados.

\section{Apresentação e Discussão dos Resultados}

Após a seleção dos artigos que tratavam sobre o tema "rankings universitários" foram identificados os indicadores utilizados e que contribuem para a classificação das universidades mundiais, conforme apresentado no Quadro 2.

Quadro 2 - Indicadores utilizados pelos Rankings Universitários Internacionais

\begin{tabular}{|c|c|c|}
\hline Ranking & Indicadores e/ou Pesos & Autores \\
\hline 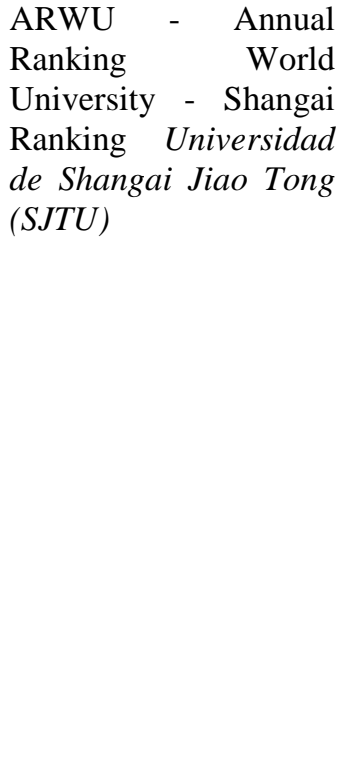 & $\begin{array}{l}\text { Alunos e pesquisadores ganhadores do prêmio Nobel } \\
(10 \%) \text {; Grupos pertencentes a instituições ganhadoras de } \\
\text { Prêmio Nobel (20\%); Pesquisadores altamente citados } \\
(20 \%) \text {; Artigos publicados na revista Nature \& Science } \\
(20 \%) \text {; Artigos que estejam indexados em algum índice de } \\
\text { citações da área de ciências e ciências sociais (20\%); } \\
\text { Acadêmicos e professores de apoio em tempo integral } \\
(10 \%) \text {. }\end{array}$ & $\begin{array}{c}\text { Aguillo et al. (2010); } \\
\text { Baldock (2013); Baty } \\
\text { (2013); Bernardino e } \\
\text { Marques (2010); } \\
\text { Bornmann, Anegõn e } \\
\text { Mutz (2013); } \\
\text { Bornmann, Mutz e } \\
\text { Daniel (2013); Buela- } \\
\text { Casal et al. (2007); } \\
\text { Charon e Wauters } \\
\text { (2008); Florian (2007); } \\
\text { Jeremic e Milenkovic } \\
\text { (2014); Marginson } \\
\text { (2014); Saisana, } \\
\text { D'Hombres e Saltelli } \\
\text { (2011); Seoane (2009); } \\
\text { Théry (2009); Turner } \\
\text { (2013); Lindblad } \\
\text { (2008) }\end{array}$ \\
\hline $\begin{array}{l}\text { CHE Excellence } \\
\text { Ranking - Centro para } \\
\text { Desenvolvimento das } \\
\text { Universidades } \\
\text { Alemanha }\end{array}$ & $\begin{array}{l}\text { Número de publicações; Número de citações (comparado } \\
\text { com o padrão mundial); Número de pesquisadores } \\
\text { frequentemente citados e participação no programa Marie } \\
\text { Curie da União Européia. }\end{array}$ & $\begin{array}{l}\text { Seoane (2009); } \\
\text { Soh (2012) }\end{array}$ \\
\hline $\begin{array}{l}\text { MINAS ParisTech - } \\
\text { École des Mines de } \\
\text { Paris }\end{array}$ & $\begin{array}{l}\text { Número de alunos como Chief Executive Officers (CEO) } \\
\text { nas } 500 \text { empresas líderes no mundo, eleitas pela revista } \\
\text { Fortune. }\end{array}$ & $\begin{array}{l}\text { Seoane (2009); } \\
\text { Théry (2009) }\end{array}$ \\
\hline $\begin{array}{llr}\text { America's } & \text { Best } \\
\text { College Review } & \text { - US } \\
\text { News and } \text { World } \\
\text { Repport }\end{array}$ & $\begin{array}{l}\text { Avaliação por pares (25\%); Retenção }(20 \% \text { para as } \\
\text { universidades nacionais e faculdades de artes liberais } \\
25 \%) \text {; Recursos do corpo docente }(20 \%) \text {; Seleção de } \\
\text { alunos }(15 \%) \text {; Recursos financeiros }(10 \%) \text {; Desempenho }\end{array}$ & $\begin{array}{l}\text { Seoane (2009); Buela- } \\
\text { Casal et al. (2007); } \\
\text { Marginson (2009); } \\
\text { Baldock (2013); }\end{array}$ \\
\hline
\end{tabular}

Valmorbida, S. M. I.; Cardoso, T. L.; Ensslin, S. R. 


\begin{tabular}{|c|c|c|}
\hline & na graduação (5\%); Recursos por alunos $(5 \%)$. & \\
\hline $\begin{array}{l}\text { THE - Times Higher } \\
\text { Education } \\
\text { University Ranking }\end{array}$ & $\begin{array}{l}\text { Revisão por pares (reputação) (40\%); Produtividade em } \\
\text { pesquisa (frequência com que os artigos da instituição são } \\
\text { citados por outros pesquisadores) }(20 \%) \text {; Qualidade } \\
\text { acadêmica (relação professor/aluno) }(20 \%) \text {; Classificações } \\
\text { de empregadores das instituições cujos diplomados } \\
\text { preferem contratar }(10 \%) \text {; Proporção de funcionários } \\
\text { estrangeiros que vêm de outros países }(5 \%) \text {; Proporção de } \\
\text { estudantes estrangeiros }(5 \%) \text {. }\end{array}$ & $\begin{array}{c}\text { Jeremic e Milenkovic } \\
\text { (2014); Marginson } \\
\text { (2014); Saisana, } \\
\text { D'Hombres e Saltelli } \\
\text { (2011); Aguillo et al. } \\
\text { (2010); Bernardino e } \\
\text { Marques (2010); } \\
\text { Bowman e Bastedo } \\
\text { (2011); Charon e } \\
\text { Wauters (2008); Théry } \\
\text { (2009); Seoane (2009); } \\
\text { Baldock (2013); Buela- } \\
\text { Casal } \text { et al. (2007) }\end{array}$ \\
\hline $\begin{array}{l}\text { Quacquarelli Symonds } \\
\text { (QS) World University } \\
\text { Rankings }\end{array}$ & $\begin{array}{l}\text { Reputação acadêmica (30\%); Reputação do empregador } \\
(10 \%) \text {; relação professor/aluno ( } 20 \%) \text {; citações por artigo } \\
(15 \%) \text {; Artigos publicados por universidade (15\%); } \\
\text { proporção de corpo docente internacional, os estudantes } \\
\text { internacionais, estudantes de intercâmbio de entrada e } \\
\text { estudantes de intercâmbio de saída ( } 2,5 \% \text { cada). }\end{array}$ & $\begin{array}{l}\text { Baldock (2013) } \\
\text { Huang (2012) } \\
\text { Jeremic e Milenkovic } \\
\quad(2014) \\
\text { Marginson (2014) } \\
\text { Rodionov, Rudskaia e } \\
\text { Kushneva (2014) }\end{array}$ \\
\hline $\begin{array}{lr}\text { Ranking } & \text { das } \\
\text { universidades } & \text { da } \\
\text { Austrália } & \text { Committee } \\
(\text { AVCC }) & \text { e } \\
\text { Universidade de } & \text { Notre } \\
\text { Dame } & \end{array}$ & $\begin{array}{l}\text { Desempenho acadêmico nas disciplinas ofertadas pela } \\
\text { universidade; publicações e citações; reconhecimento } \\
\text { prova pericial (a escolha do corpo de ensino e pesquisa } \\
\text { prêmios recebidos); Qualidade de graduados graduandos; a } \\
\text { inserção no mercado de trabalho e satisfação dos alunos. }\end{array}$ & Seoane (2009) \\
\hline $\begin{array}{lr}\text { College } & \text { Ranking } \\
\text { Reformed } & -N S S E \\
\text { (National Survey of } \\
\text { Students Engagement) }\end{array}$ & $\begin{array}{l}\text { Mérito acadêmico; experiências educacionais de interação } \\
\text { da universidade com o serviço à comunidade; inserção dos } \\
\text { alunos no contexto: econômico, social, racial e étnico; } \\
\text { ambiente do campus; valor agregado/ crescimento do } \\
\text { aluno; finalização de projetos; índice de retenção e } \\
\text { graduação de alunos; conclusão do curso e entrada no } \\
\text { mercado de trabalho; satisfação do aluno; educação: } 20 \% \text {; } \\
\text { aprendizagem: } 30 \% \text {; retenção e graduação: } 20 \% \text {; sucesso } \\
\text { na vida pós-escola: } 30 \% \text {. }\end{array}$ & Seoane (2009) \\
\hline $\begin{array}{lr}\text { Canadian } & \text { Education } \\
\text { Report } & \text { Series } \\
\text { Educational } & \text { Policy } \\
\text { Institute (EPI) } & \end{array}$ & $\begin{array}{l}\text { Atributos e habilidades dos alunos no início do curso; } \\
\text { recursos financeiros e materiais disponíveis para } \\
\text { professores e alunos para fins educacionais que favorecem } \\
\text { a aprendizagem: número de professores, tempo que os } \\
\text { alunos têm de estar em contato com os professores, e tipos } \\
\text { de exames enfrentados; retorno sobre aprendizagem: } \\
\text { pensamento crítico, habilidades de raciocínio analítico e } \\
\text { experiência, e retenção e ao final do curso; sistema } \\
\text { educacional: taxas de emprego e renda, a satisfação no } \\
\text { trabalho. }\end{array}$ & Seoane (2009) \\
\hline $\begin{array}{l}\text { CWTS- Leiden } \\
\text { Ranking - Ranking da } \\
\text { Universidade de } \\
\text { Leiden }\end{array}$ & $\begin{array}{l}\text { Volume de trabalhos científicos; volume de citações } \\
\text { desses artigos; citações por artigo, o número de artigos no } \\
\text { top } 10 \% \text { de sua área por taxas de citação; a proporção dos } \\
\text { artigos da universidade na Thomson-ISI Web of } \\
\text { Knowledge. }\end{array}$ & $\begin{array}{l}\text { Aguillo et al. (2010); } \\
\text { Bornmann, Anegõn e } \\
\text { Mutz (2013); } \\
\text { Bornmann, Mutz e } \\
\text { Daniel (2013); } \\
\text { Marginson (2014); }\end{array}$ \\
\hline $\begin{array}{l}\text { Scimago Scopus } \\
\text { Scopus Elsevier }\end{array}$ & $\begin{array}{l}\text { Número total de documentos publicados em revistas } \\
\text { acadêmicas indexadas na Base Scopus. }\end{array}$ & $\begin{array}{l}\text { Bornmann, Anegõn e } \\
\text { Mutz (2013); De } \\
\text { Mesnard (2012); } \\
\text { Marginson (2014); } \\
\text { Jeremic e Milenkovic } \\
\quad \text { (2014) }\end{array}$ \\
\hline $\begin{array}{l}U \text {-Multirank ou } U \text { - } \\
\text { Map }\end{array}$ & $\begin{array}{l}\text { Reputação acadêmica e satisfação do aluno; níveis e } \\
\text { orientações de graus, faixa de assunto, perfil do estudante, }\end{array}$ & $\begin{array}{l}\text { Boulton (2011); } \\
\text { Erkkilä (2014); }\end{array}$ \\
\hline
\end{tabular}




\begin{tabular}{|c|c|c|}
\hline & $\begin{array}{l}\text { atividade de pesquisa, troca de conhecimento, orientação } \\
\text { internacional e envolvimento regional. }\end{array}$ & Marginson (2014) \\
\hline $\begin{array}{lr}\text { Asia's } & \text { Best } \\
\text { Universities Ranking }\end{array}$ & $\begin{array}{l}\text { Opinião de especialistas (20\%); número de alunos do } \\
\text { primeiro ano e aceites em comparação com o total de } \\
\text { candidatos; numero de matriculados em comparação com } \\
\text { os alunos aceitos; pontuação média dos alunos do primeiro } \\
\text { ano no teste de entrada da universidade (25\%); } \\
\text { infraestrutura: professores em tempo integral / } \\
\text { pesquisadores com doutorado; professores em tempo } \\
\text { integral / pesquisadores com mestrado; salário médio; } \\
\text { gastos da universidade por professor; taxa aluno/professor } \\
\text { (25\%); citações em revistas acadêmicas indexadas na Web } \\
\text { of Science; artigos em revistas e jornais; trabalhos } \\
\text { apresentados em conferências internacionais; livros } \\
\text { publicados; incentivo/financiamento da pesquisa, número } \\
\text { de estudantes de pós-graduação (20\%); recursos } \\
\text { financeiros: gasto total por aluno; gastos com a biblioteca } \\
\text { por aluno; tamanho da banda de internet; computadores } \\
\text { disponíveis ao público e pontos de conexão; os gastos de } \\
\text { laboratório, apenas para a ciência e escolas de tecnologia } \\
\text { (10\%); }\end{array}$ & $\begin{array}{l}\text { Buela-Casal et al. } \\
\text { (2007); }\end{array}$ \\
\hline $\begin{array}{l}\text { Excellence French and } \\
\text { German universities }\end{array}$ & $\begin{array}{l}\% \text { de estudantes estrangeiros; \% de docentes com grau de } \\
\text { doutor; } \% \text { de estudos de pós-graduação (oficial mestrado e } \\
\text { doutorado); } \% \text { de alunos de pós-graduação (inscritos em } \\
\text { mestrado e } \mathrm{PhD} \text { oficial). }\end{array}$ & $\begin{array}{l}\text { Benito e Romera } \\
\text { (2011) }\end{array}$ \\
\hline $\begin{array}{l}\text { HEEACT Performance } \\
\text { Ranking of Scientific } \\
\text { Papers for World } \\
\text { Universities - Higher } \\
\text { Education Evaluation } \\
\text { and Accreditation } \\
\text { Council of Taiwan }\end{array}$ & $\begin{array}{l}\text { Número de publicações e citações de acordo com as bases } \\
\text { de dados de citação Thomson ISI (Science Citation Index, } \\
\text { Science Citation Index Social Essential Science } \\
\text { Indicators), dando especial atenção às publicações } \\
\text { recentes. }\end{array}$ & $\begin{array}{l}\text { Soh (2012); } \\
\text { Aguillo et al. (2010); } \\
\text { Lin, Huang e Chen } \\
\text { (2013); Marginson } \\
\text { (2009); }\end{array}$ \\
\hline $\begin{array}{l}\text { International } \\
\text { Champions League of } \\
\text { Research Institutions }\end{array}$ & $\begin{array}{l}\text { Indicadores por disciplinas: número de artigos na base de } \\
\text { dados Web of Science; fator de impacto das publicações; } \\
\text { atividades de publicação; apresentação de publicações. } \\
\text { Indicadores da instituição: grau de especialização de } \\
\text { publicações; total de publicações. }\end{array}$ & $\begin{array}{l}\text { Buela-Casal et al. } \\
\text { (2007) }\end{array}$ \\
\hline $\begin{array}{ll}\text { Spanish } & I-U G R \\
\text { Rankings } & \end{array}$ & $\begin{array}{l}\text { Publicações indexadas na Web of Science (SCI, SSCI e A } \\
\& \text { CI); rede de relacionamento entre pesquisadores; } \\
\text { coeficiente de Gini é um indicador de desigualdade que } \\
\text { mostra a concentração ou dispersão das distribuições; } \\
\text { Número de disciplinas. }\end{array}$ & $\begin{array}{c}\text { Robinson-García et al } \\
\text { (2014) }\end{array}$ \\
\hline Portuguese Ranking & $\begin{array}{l}\text { Pesquisas publicadas; \% aluno/professor; taxa de evasão; } \\
\% \text { de estudantes estrangeiros; \% de funcionários } \\
\text { estrangeiros; taxa de empregabilidade; média do primeiro } \\
\text { salário; percentual do faturamento; }\end{array}$ & $\begin{array}{l}\text { Bernardino e Marques } \\
\text { (2010) }\end{array}$ \\
\hline $\begin{array}{l}\text { Webometrics (Ranking } \\
\text { Web of Universities) }\end{array}$ & $\begin{array}{l}\text { Número de páginas indexadas no Google, no Yahoo e no } \\
\text { Bing; número de indexação no Yahoo inverse links; } \\
\text { número de arquivos (tipos PDF, DOC, PPT, e PPS) } \\
\text { indexados pelo Google, Yahoo e Bing; número de } \\
\text { documentos escolares indexados pelo Google Scholar. }\end{array}$ & $\begin{array}{c}\text { Soh (2012); Théry } \\
\text { (2009); Hsieh et al. } \\
\text { (2012); Aguillo et al. } \\
\text { (2010); Marginson } \\
\text { (2009); Stergiou e } \\
\text { Lessinich (2013) }\end{array}$ \\
\hline $\begin{array}{lr}\text { Ranking } & \text { das } \\
\text { Universidades r de } & \\
\text { Pesquisa da América } & - \\
\text { Ther } & \text { Lombardi } \\
\text { Program } & \end{array}$ & $\begin{array}{l}\text { Gasto total com pesquisa; gasto federal em pesquisa; } \\
\text { alocação de recursos; doações anuais; número de membros } \\
\text { da universidade em entidades nacionais; reconhecimento } \\
\text { da universidade; número de doutores; selecionados pós- } \\
\text { doutorado; alunos ingressantes. }\end{array}$ & Seoane (2009) \\
\hline
\end{tabular}

Fonte: Dados da Pesquisa (2015).

Valmorbida, S. M. I.; Cardoso, T. L.; Ensslin, S. R. 
Percebe-se que, os indicadores mais frequentes nos rankings são: reputação da universidade/aceitação dos alunos pelo mercado; artigos da instituição publicados (45\%); artigos da instituição indexados a bases de dados (40\%); número de artigos da instituição citados por outros pesquisadores e infraestrutura financeira, administrativa e de pessoal (35\%), número de pesquisadores da instituição citados por outros trabalhos, relação professor/aluno (30\%); indicadores de desempenho acadêmico, retenção e conclusão, abertura para estudantes estrangeiros e empregar estrangeiros (25\%); satisfação dos alunos (20\%). Embora tenham sido utilizados por vários trabalhos, não se pode afirmar que esses indicadores representem a mensuração do mesmo objetivo, haja vista que, muitos indicadores são apresentados de forma ambígua, podendo ser avaliados de forma diferente e/ou subjetiva, não sendo possível sua replicabilidade.

Observa-se que, alguns indicadores foram utilizados exclusivamente por um único ranking, tais como salário de docente; Coeficiente de Gini; avaliação dos cursos; número de alunos ingressantes; indexação nos sites de busca como o Google; gastos com pesquisa; valor da receita por aluno; alocação de recursos. Assim, como ocorrem com os indicadores mais utilizados, estes são de difícil rastreabilidade, haja vista não especificarem a fonte dos dados.

Constatou-se ainda que, a maioria dos rankings utiliza indicadores diretamente relacionados com a pesquisa, em detrimento das demais áreas da instituição. Dessa forma, falham ao tentar medir a qualidade institucional, tendo em vista que, outros aspectos importantes influenciam na qualidade do ensino (ROBINSON, 2013).

Quando usados para avaliar cursos, há predominância na avaliação das áreas das chamadas ciências exatas e biológicas (biologia, química, física e matemática), não prestigiando outras áreas como as ciências sociais, conforme apontado por Charon e Wauters (2008) e Seoane (2009).

Como esses rankings são muitas vezes utilizados como ferramenta de marketing pelas universidades para mostrar sua excelência educacional ou de pesquisa, há necessidade de fornecer rankings tão precisos quanto possível (JEREMIC; MILENKOVIC, 2014) que representem adequadamente todas as atividades da instituição (LIN; HUANG; CHEN, 2013). Por isso, os indicadores são tão importantes, à medida que podem tornar-se uma propaganda negativa, caso esses indicadores não demonstrem transparência e replicabilidade (BENITO; ROMERA, 2011; FLORIAN, 2007).

E, por fim, cabe salientar que, conforme apontam Benito e Romera (2011, p. 19) cada sistema de classificação/ranqueamento deve indicar claramente os objetivos pelo qual foi construído e identificar com precisão o público abordado, e construir indicadores alinhados com o objetivo do ranking e aos interesses do público a que se destina ou que será influenciado por ele. Dessa forma, podem atuar para melhorar seu desempenho, tornando-se assim ferramentas úteis para os interessados.

\subsection{Contribuições da Pesquisa para o Conhecimento Científico}

A partir da análise dos resultados encontrados percebem-se gaps que podem promover avanços nesta área de conhecimento se forem investigados, quais sejam:

- Eliminar ambiguidade nos indicadores/métricas utilizados, a fim de permitir a rastreabilidade dos dados e garantir credibilidade no resultado apurado;

- Incluir indicadores que privilegiem avaliar a qualidade de todas as àreas da instituição, quais sejam: ensino, pesquisa, extensão, gestão, dentre outras;

- Necessidade de estabelecimento de pesos e formas adequados de agregação para somatória dos indicadores desenvolvidos;

- Necessidade de desenvolvimento de metodologia capaz de 'lidar' com indicadores e métricas de mensuração que neutralizem a característica de 
estágio de maturidade (porte, idade, finalidade, recursos e culturas diferentes) das universidades. Haja vista que, da forma que estão construídos os indicadores e configurados os rankings claramente privilegiam instituições mais antigas, com mais recursos e investimentos;

- A análise dos indicadores mostrou que não são claras as métricas adotadas por rankings, ou seja, poucos identificam os objetivos e indicadores claramente estabelecidos para esclarecer o que está sendo mensurado, culminando em dificuldades de utilização desta informação para melhoria do desempenho apresentado e por conta disso, tem gerado críticas na utilização dos rankings;

- Construção de metodologias de ranqueamento que sejam capazes de se comunicar com todos os stakeholders sobre o desempenho e oferecer estratégias para acionar melhor desempenho, promovendo, assim, a atividade de gestão universitária;

- Desenvolvimento de estudos prospectivos que indiquem o desempenho das universidades nos rankings em cenários futuros, ao invés de estudos baseados em dados passados; e,

- Desenvolver sistemas de avaliação de desempenho para orientar as universidades a se autogerenciarem para que, utilizando as informações possam agir para melhorar sua posição no ranking.

$\mathrm{Na}$ percepção dos autores deste trabalho, a informação gerada pelos indicadores e rankings universitários, da forma que estão delineados, pouco contribui para a atividade de gestão universitária e, não conseguem gerar informações que possam promover a aprendizagem organizacional.

E, por fim, percebe-se que a avaliação de desempenho, voltada aos rankings universitários, pode oferecer muitos benefícios para a gestão de instituições de ensino e pesquisa; no entanto, pela análise realizada, percebe-se que as universidades não estão conseguindo fazer uso das infromações necessárias para apoiar sua gestão.

\section{Considerações Finais}

Este artigo identificou os indicadores utilizados pelos rankings universitários internacionais. Fez uso do instrumento ProKnow-C, para, de forma estruturada, formar um portfólio bibliográfico representativo do tema "rankings universitários", composto por 32 artigos internacionais. Com base nesse portfólio, foram identificados os vários rankings existentes com seus respectivos indicadores.

Foram observados 19 rankings construídos para diversas finalidades ao redor do mundo. Os indicadores mais frequentes desses rankings são relacionados com a infraestrutura e a reputação da universidade, dados bibliométricos, tais como artigos publicados, número de citações de artigos e de pesquisadores e artigos indexados nas bases de dados.

Constatou-se também que, há muitas diferenças de construção de indicadores e atribuição de pesos utilizados pelos rankings; no entanto, cabe salientar que a partir da finalidade dos rankings é que devem ser construídos os indicadores correlacionados com as necessidades de informação dos usuários para o qual o ranking foi idealizado.

$\mathrm{Na}$ percepção das autoras deste trabalho, a informação gerada pelos indicadores e rankings universitários, da forma que estão delineados, pouco contribui para a atividade de gestão universitária tanto em curto quanto em longo prazo; haja vista que, não conseguem gerar informações que possam promover a aprendizagem organizacional.

E, por fim, percebe-se que a avaliação de desempenho pode oferecer muitos benefícios para a gestão de instituições de ensino e pesquisa, voltadas aos rankings universitários, no 
entanto, pela análise realizada, percebe-se que a literatura analisada não está explorando estes benefícios para contribuir com a atividade de gestão das instituições de ensino superior.

Este artigo limitou-se à identificação e análise dos indicadores expostos nos artigos do portfólio bibliográfico representativo do tema "rankings universitários". Sugere-se para futuras pesquisas a análise crítica da identificação e das métricas dos objetivos e indicadores utilizados pelos rankings.

\section{Referências}

AGUILlO, I. F.; BAR-ILAN, J.; LEVENE, M.; ORTEGA, J. L. Comparing university rankings. Scientometrics, v. 85, n. 1, p. 243-256, 2010.

BALDOCK, C. University rankings and medical physics. Australasian Physical and Engineering Sciences in Medicine, v. 36, n. 4, p. 375-378, 2013.

BATY, P. The times higher education world university rankings, 2004-2012. Ethics in Science and Environmental Politics, v. 13, n. 2, p. 125-130, 2013.

BENITO, M.; ROMERA, R. Improving quality assessment of composite indicators in university rankings: A case study of French and German universities of excellence. Scientometrics, v. 89, n. 1, p. 153-176, 2011.

BERNARDINO, P.; MARQUES, R. C. Academic rankings: An approach to rank portuguese universities. Ensaio, v. 18, n. 66, p. 29-48, 2010.

BORNMANN, L.; DE MOYA ANEGÕN, F.; MUTZ, R. Do universities or research institutions with a specific subject profile have an advantage or a disadvantage in institutional rankings?: A Latent Class Analysis with Data from the SCImago Ranking. Journal of the American Society for Information Science and Technology, v. 64, n. 11, p. 2310-2316, 2013.

BORNMANN, L.; MUTZ, R.; DANIEL, H. D. Multilevel-statistical reformulation of citation-based university rankings: The Leiden ranking 2011/2012. Journal of the American Society for Information Science and Technology, v. 64, n. 8, p. 1649-1658, Aug 2013.

BOULTON, G. University rankings: Diversity, excellence and the European initiative. Procedia - Social and Behavioral Sciences, v. 13, p. 74-82, 2011.

BOWMAN, N. A.; BASTEDO, M. N. Anchoring effects in world university rankings: Exploring biases in reputation scores. Higher Education, v. 61, n. 4, p. 431-444, 2011.

BUELA-CASAL，G.; GUTIÉRREZ-MARTÍNEZ，O.; BERMÚDEZ-SÁNCHEZ，M. P.; VADILLO-MUÑOZ, O. Comparative study of international academic rankings of universities. Scientometrics, v. 71, n. 3, p. 349-365, 2007.

CHARON, A.; WAUTERS, J. P. University ranking: a new tool for the evaluation of higher education in Europe. Nephrology Dialysis Transplantation, v. 23, n. 1, p. 62-64, Jan. 2008. 
CHENG, Y.; LIU, N. C. Academic ranking of world universities by broad subject fields. Higher Education in Europe, v. 32, n. 1, p. 17-29, 2007.

CRESWELL, J. W. Research Design: Qualitative, Quantitative, and Mixed Methods Approaches. 4 th ed. SAGE Publications, Inc, 2014.

DE MESNARD, L. On some flaws of university rankings: The example of the SCImago report. Journal of Socio-Economics, v. 41, n. 5, p. 495-499, 2012.

DILL, D. D.; SOO, M. Academic quality, league tables, and public policy: A cross-national analysis of university ranking systems. Higher Education, v. 49, n. 4, p. 495-533, Jun. 2005.

DUTRA, A.; RIPOLL-FELIU, V.M.; FILLOL, A. G.; ENSSLIN, S. R.; ENSSLIN, L. The construction of knowledge from the scientific literature about the theme seaport performance evaluation, International Journal of Productivity and Performance Management, v. 64, iss 2 , p. $243-269,2015$.

ENSSLIN, L.; ENSSLIN, S. R.; PINTO, H. M.; Processo de investigação e Análise bibliométrica: Avaliação da Qualidade dos Serviços Bancários. RAC, v. 17, n. 3, p. 325-349, 2013.

ENSSLIN, S. R.; RIPOLL-FELIU, V. M.; ENSSLIN, L.; DUTRA, A. Performance Evaluation to Support the University Management Activity. La Pensée (Paris), v. 76, p. 2-17, 2014a.

ENSSLIN, S. R.; ENSSLIN, L.; IMLAU, J. M.; CHAVES, L. C.. Processo de mapeamento das publicações científicas de um tema: portfólio bibliográfico e análise bibliométrica sobre avaliação de desempenho de cooperativas de produção agropecuária. Revista de Economia e Sociologia Rural (Impresso), v. 52, p. 587-608, 2014 b.

ERKKILÄ, T. Global university rankings, transnational policy discourse and higher education in Europe. European Journal of Education, v. 49, n. 1, p. 91-101, 2014.

FLORIAN, R. V. Irreproducibility of the results of the Shanghai academic ranking of world universities. Scientometrics, v. 72, n. 1, p. 25-32, 2007.

HSIEH, S. M.; LO, S. A.; HSU, C. C.; LIN, W. C. Novel cloud service for improving world universities ranking. In: Proceedings of the 9th International Conference on Information Technology, ITNG 2012, 2012. Anais Eletrônicos., 2012. Disponível em: < http://www.scopus.com/inward/record.url?eid=2-s2.084863931111\&partnerID=40\&md5=7ff87c300e76ea10dca6b9d5fca60326 >.

HUANG, M. H. Opening the black box of QS world university rankings. Research Evaluation, v. 21, n. 1, p. 71-78, 2012.

IOANNIDIS, J. P. A.; PATSOPOUlOS, N. A.; KAVVOURA, F. K.; TATSIONI, A.; EVANGELOU, E.; KOURI, I.; CONTOPOULOS-IOANNIDIS, D. G.; LIBEROPOULOS, G. International ranking systems for universities and institutions: A critical appraisal. BMC Medicine, v. 5, 2007. 
JEREMIC, V.; MILENKOVIC, M. J. Evaluation of Asian university rankings: Position and perspective of leading Indian higher education institutions. Current Science, v. 106, n. 12, p. 1647-1653, 2014.

KEHM, B. M. Global university rankings - Impacts and unintended side effects. European Journal of Education, v. 49, n. 1, p. 102-112, 2014.

LACERDA, R. T. O.; ENSSLIN, L.; ENSSLIN, S. R. Uma Análise Bibliométrica da literatura sobre Estratégia e Avaliação de Desempenho. Gestão \& Produção, v. 19, n. 1, p. 59-78, 2012.

LIN, C. S.; HUANG, M. H.; CHEN, D. Z. The influences of counting methods on university rankings based on paper count and citation count. Journal of Infometrics, v. 7, n. 3, p. 611621, 2013.

LINDBLAD, S. Navigating in the field of University positioning: On international ranking lists, quality indicators and higher education governing. European Educational Research Journal, v. 7, n. 4, p. 438-450, 2008.

LUKMAN, R.; KRAJNC, D.; GLAVIC, P. University ranking using research, educational and environmental indicators. Journal of Cleaner Production, v. 18, n. 7, p. 619-628, May 2010 .

MARGINSON, S. Open source knowledge and university rankings. Thesis Eleven, v. 96, n. 1, p. 9-39, 2009.

. University rankings and social science. European Journal of Education, v. 49, n. 1, p. 45-59, 2014.

PUSSER, B.; MARGINSON, S. University rankings in critical perspective. Journal of Higher Education, v. 84, n. 4, p. 544-568, 2013.

ROBINSON, D. The mismeasure of higher education? The corrosive effect of university rankings. Ethics in Science and Environmental Politics, v. 13, n. 2, p. 11-17, 2013.

ROBINSON-GARCÍA，N.; TORRES-SALINAS, D.; DELGADO LÓPEZ-CÓZAR, E.; HERRERA, F. An insight into the importance of national university rankings in an international context: the case of the I-UGR rankings of Spanish universities. Scientometrics, 2014.

RODIONOV, D. G.; RUDSKAIA, I. A.; KUSHNEVA, O. A. The importance of the university world rankings in the context of globalization. Life Science Journal, v. 11, n. 10 SPEC. ISSUE, p. 442-446, 2014.

ROSA, F. S. da; ENSSLIN, S. R.; ENSSLIN, L.; LUNKES, R. J. Gestão da evidenciação ambiental: um estudo sobre as potencialidades e oportunidades do tema. Eng. Sanit. Ambient. [online], v. 16, n. 2, p. 157-166, 2011.

SAISANA, M.; D'HOMBRES, B.; SALTELLI, A. Rickety numbers: Volatility of university rankings and policy implications. Research Policy, v. 40, n. 1, p. 165-177, 2011. 
SEOANE, A. Y. ¿Cómo se evalúan las universidades de clase mundial? Revista de la educación superior, v. 38, n. 150, p. 113-120, 2009.

SILVA, R. V.; ENSSLIN, S. R.; RIPOLL-FELIU, V.; SOLER, C. C.. E-government and Public Accounting Information: Bibliometric and Systemic Analysis. International Research Journal of Finance and Economics, Issue 122, 2014.

$\mathrm{SOH}, \mathrm{K}$. World university rankings: What is in for top ten East Asian universities? New Horizons in Education, v. 60, n. 2, 2012.

STERGIOU, K. I.; LESSENICH, S. On impact factors and university rankings: From birth to boycott. Ethics in Science and Environmental Politics, v. 13, n. 2, p. 101-111, 2013.

TASCA, J. E.; ENSSLIN, L.; ENSSLIN, S. R.; ALVES, M. B. M. An approach for selecting a theoretical framework for the evaluation of training programs. Journal of European Industrial Training, v.34, n. 7, p. 631-655, 2010.

THÉRY, H. The academic ranking of world universities: Shanghai and the others. Mappemonde, v. 96, n. 4, 2009.

TURNER, D. A. World class universities and international rankings. Ethics in Science and Environmental Politics, v. 13, n. 2, p. 167-176, 2013. 